\title{
Eculizumab safely reverses neurologic impairment and eliminates need for dialysis in severe atypical hemolytic uremic syndrome
}

This article was published in the following Dove Press journal:

Clinical Pharmacology:Advances and Applications

23 May 2011

Number of times this article has been viewed

\author{
Maro Ohanian \\ Christian Cable \\ Kathleen Halka \\ Department of Hematology \\ and Oncology, Scott and White \\ Healthcare, The Texas A\&M \\ Health Science Center College \\ of Medicine, Temple, TX, USA
}

Correspondence: Maro Ohanian Department of Hematology and Oncology, Scott and White Healthcare, 240I South 3 I st Street, Temple, TX 76508, USA

Tel +I 28I 217 I974

Fax + I 254 77| 4890

Email ohanianus@yahoo.com

\begin{abstract}
This case report describes how eculizumab reversed neurologic impairment and improved renal damage in severe atypical hemolytic uremic syndrome. A 50-year-old female, after presenting with diarrhea and abdominal pain, developed pancolitis, acute renal failure, and thrombocytopenia. The patient underwent total abdominal colectomy. Pathology confirmed ischemic colitis with scattered mesenteric microthrombi. Due to mental and respiratory decline, she remained intubated. Continuous venovenous hemodialysis was initiated. Renal failure, neurologic changes, hemolysis, thrombotic microangiopathy, and low complement levels all suggested atypical hemolytic uremic syndrome. Eculizumab $900 \mathrm{mg}$ was administered intravenously on hospital day 6 and continued weekly for four doses followed by maintenance therapy. She recovered neurologically and renally after the third dose, and hematologically by the sixth dose. Her recovery has been sustained on long-term eculizumab treatment. In severe atypical hemolytic uremic syndrome, eculizumab safely reverses neurologic impairment and eliminates the need for dialysis. The optimal duration of treatment with eculizumab remains to be determined.
\end{abstract}

Keywords: eculizumab, thrombotic microangiopathy, atypical hemolytic uremic syndrome

\section{Introduction}

Atypical hemolytic uremic syndrome involves congenital or acquired dysregulation of the alternative complement pathway. Uncontrolled chronic systemic complement activation causes inflammation and thrombotic microangiopathy. Dysfunctional alternative complement pathway activation reduces host cell protection and results in inappropriate complement activation of platelets and endothelial cells. ${ }^{1,2}$ Atypical hemolytic uremic syndrome can cause multiorgan failure and severe neurologic changes. We present a case of severe atypical hemolytic uremic syndrome with significant neurologic damage amidst multiorgan failure, including severe renal failure. Terminal complement inhibition with eculizumab safely reversed the neurologic changes and eliminated the need for dialysis. While eculizumab is not yet approved by the US Food and Drug Administration for atypical hemolytic uremic syndrome, this case report is one of several illustrating its safety and efficacy.

\section{Case report}

A fifty-year-old female with a history of rheumatoid arthritis was transferred to our intensive care unit with sepsis, pancolitis, acute renal failure, and thrombocytopenia. Three days prior to transfer, she had presented with malaise, bloody diarrhea, and 
abdominal pain. Her home medications included an estrogen patch, lansoprazole, and twice-weekly etanercept. Stool studies, including tests for Escherichia coli 0157:H7 and Clostridium difficile, were all negative. Prior to transfer, she was on ceftriaxone, ciprofloxacin, and dexamethasone for colitis. At our facility, she remained on broad-spectrum antimicrobials, including fluconazole, ciprofloxacin, metronidazole, and pipercillin-tazobactum. Because of her sepsis, she remained on adrenal support with methylprednisolone.

Her initial laboratory investigations revealed a count of $12.7 \mathrm{gm} / \mathrm{dL}$ hemoglobin, white blood cells of $31.4 \times 10 \mathrm{e} 9 / \mathrm{L}$, creatinine of $3.8 \mathrm{mg} / \mathrm{dL}$, and platelets of $28 \times 10 \mathrm{e} / \mathrm{L}$. Coagulation studies did not suggest disseminated intravascular coagulation. Aspartate aminotransferase was elevated at $118 \mathrm{U} / \mathrm{L}$. Alanine aminotransferase was normal. Lactate dehydrogenase was 1199 units per liter (U/L). Haptoglobin was less than $6 \mathrm{mg} / \mathrm{dL}$. Direct Coomb's test was negative. Chest x-ray revealed bibasilar opacities and small bilateral pulmonary effusions. Reticulocyte count was $2.7 \%$. Decreased complement levels were later noted: C3 was 35 (83-184) mg/ $\mathrm{dL}$ and $\mathrm{C} 4$ was 6 (17-59) mg/dL. ADAMTS13 activity was adequate at $63 \%$ (drawn prior to treatment). By hospital day 5 , there were occasional schistocytes on the peripheral smear.

On the day of transfer, she underwent total abdominal colectomy with end ileostomy. During surgery she received 2 units (U) of packed red blood cells, $2 \mathrm{U}$ of fresh frozen plasma, and $2 \mathrm{U}$ of pooled platelets. After surgery, her platelets rose, and continued to rise during the first five days of the admission, without additional transfusions. The rising platelets after colectomy suggested previous platelet consumption by a thrombotic microangiopathic process in the colon. This was later confirmed by the pathology report, which described diffuse ischemic colitis with scattered fibrin microthrombi along the mesentery, consistent with microangiopathy.

With mental and respiratory decline, she remained intubated. By hospital day 3, she did not respond to commands and was generally unresponsive to voice and painful stimuli for the next 11 days. By hospital day 4, there were reports of seizure activity. A noncontrast computed tomography scan of the brain was unremarkable; she was too unstable to leave the intensive care unit for a magnetic resonance imaging scan of the brain. Electroencephalographic findings revealed no definite epileptiform activity, but were consistent with diffuse cerebral dysfunction.

As renal failure worsened, continuous venovenous hemodialysis was initiated on hospital day 4. Her clinical picture, with severe renal failure, profound neurologic changes, severe hemolysis, thrombotic microangiopathy, and low complement levels, were all suggestive of atypical hemolytic uremic syndrome. Because atypical hemolytic uremic syndrome is characterized by chronic uncontrolled systemic complement activation, ${ }^{2}$ the anti-C5 monoclonal antibody, eculizumab, was administered intravenously at a dose of $900 \mathrm{mg}$ on hospital day 6. It was continued weekly for four doses, followed by maintenance therapy. Therapeutic plasma exchange was also administered for a few hours before her first dose of eculizumab. Because treatment with eculizumab increases the risk of infection with Neisseria meningitidis, the meningococcal vaccine was given prior to the first dose, and 14 days of ciprofloxacin was ordered for meningococcal prophylaxis. ${ }^{3}$

Four days after receiving her first dose of eculizumab, the patient's lactate dehydrogenase dropped to $837 \mathrm{U} / \mathrm{L}$ and her complement levels rose: $\mathrm{C} 3$ rose to $81(83-184) \mathrm{mg} / \mathrm{dL}$ and $\mathrm{C} 4$ rose to 16 (17-59) mg/dL, as shown in Figures 1 and 2. Seven days into treatment, she was opening her eyes, tracking, and localizing to painful stimuli. The day after her second dose, her lactate dehydrogenase decreased to $681 \mathrm{U} / \mathrm{L}$. Two days after the second dose she was nodding appropriately, following commands, and interacting with her family. Three days after the second dose, her lactate dehydrogenase decreased to $498 \mathrm{U} / \mathrm{L}$ and her dialysis frequency was reduced to three times per week. Four days after her second dose of

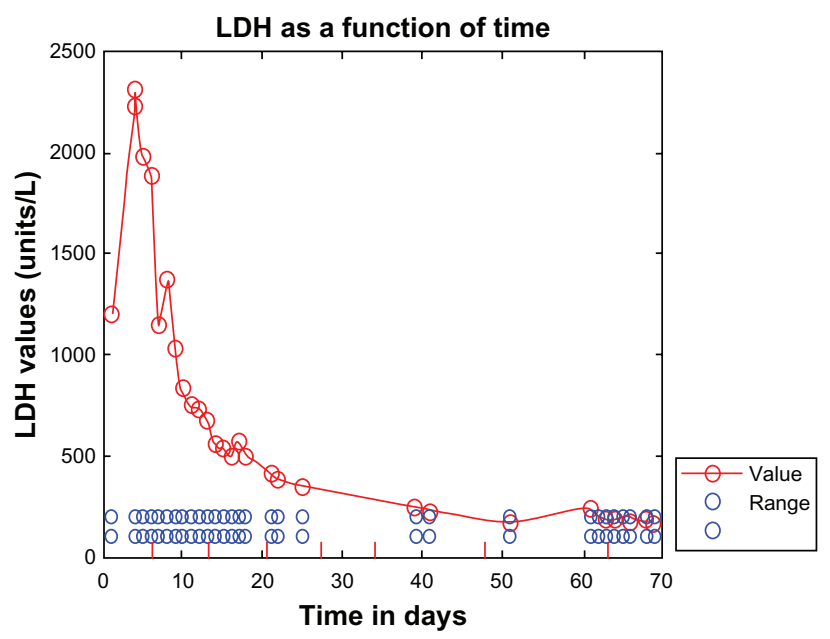

Figure I Lactate dehydrogenase (normal 108-202 U/L) shown as a function of time (hospital days). Lactate dehydrogenase gradually dropped after initiating treatment with eculizumab. Eculizumab administration is denoted by vertical tick marks (hospital days 6, 13, 20, 27, 34, 48, and day 63). Four days after the first dose, lactate dehydrogenase dropped to $837 \mathrm{U} / \mathrm{L}$. After the second dose of eculizumab on day 13, lactate dehydrogenase dropped to $556 \mathrm{U} / \mathrm{L}$. A week after the third dose, lactate dehydrogenase was down to $349 \mathrm{U} / \mathrm{L}$. Three days after the sixth dose, lactate dehydrogenase was normal. It did rise slightly before the seventh dose was due. 


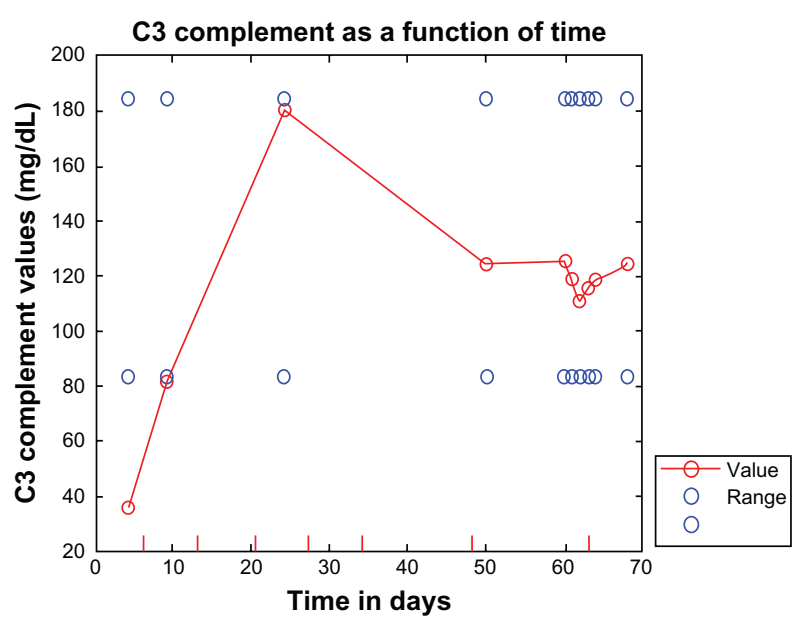

Figure $2 \mathrm{C} 3$ (normal $83-184 \mathrm{mg} / \mathrm{dL}$ ) shown as a function of time (hospital days). Eculizumab administration is denoted by vertical tick marks (hospital days 6, 13, 20, $27,34,48$, and day 63 ). After the second dose of eculizumab on day 13, C3 rose to $8 \mathrm{I}$. Thirteen days after the sixth dose, $\mathrm{C} 3$ was normal and remained normal.

eculizumab, she was ready to be weaned from the ventilator; within several days, she no longer required oxygen. A week after her third dose of eculizumab, her lactate dehydrogenase was down to $349 \mathrm{U} / \mathrm{L}$ (Figure 1).

Three days after her third dose, she was urinating up to $875 \mathrm{~mL}$, and her cognition and memory had recovered to baseline. After the fourth dose of eculizumab, her urine output reached $1451 \mathrm{~mL}$, and dialysis was discontinued. Three days after her sixth dose, her lactate dehydrogenase, haptoglobin, C3, and C4 were all normal (see Figures 1-5 for a summary of treatment responses).

This patient presented with rapidly progressive atypical hemolytic uremic syndrome characterized by multiorgan failure, including severe neurologic decline. Her renal recovery was remarkable, and she has remained off dialysis with long-term eculizumab treatment. Additionally, her creatinine has continued to decrease off dialysis, with recent levels as low as $2.24 \mathrm{mg} / \mathrm{dL}$ (see Figure 3 for a summary of creatinine over time).

While renal failure is generally the most pronounced complication of atypical hemolytic uremic syndrome, neurologic impairment can also occur in severe cases. Our patient's rapid and profound neurologic deterioration appears to have resulted from significant thrombotic microangiopathy. Despite 11 days in the intensive care unit without meaningful neurologic responses, the patient recovered total neurologic function after only a few doses of eculizumab. Her swift and complete neurologic recovery makes this case particularly noteworthy. Twenty-six days after the first dose of eculizumab, a magnetic resonance imaging brain scan was

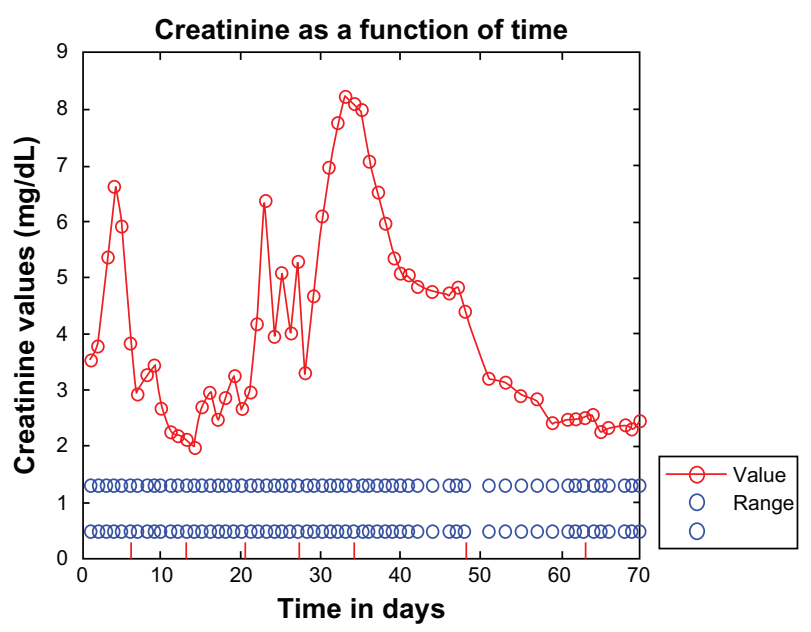

Figure 3 Creatinine (normal $0.5-1.3 \mathrm{mg} / \mathrm{dL}$ ) shown as a function of time (hospital days). Eculizumab administration is denoted with vertical tick marks (hospital days 6 , $13,20,27,34,48$, and day 63). The start and end dates of continuous venovenous hemodialysis are marked on the time axis by 2 carat $\left({ }^{\wedge}\right)$ symbols (hospital days 4 and 27). Three days after the second dose of eculizumab, dialysis frequency was reduced to three times per week. Continuous venovenous hemodialysis was completely discontinued after the fourth dose of eculizumab. Despite a delayed and reduced dose of eculizumab at the seventh administration, creatinine continued to decrease to as low as $2.24 \mathrm{mg} / \mathrm{dL}$.

performed upon request by the neurologist. The findings were consistent with a subacute right parietal lobe infarction with areas of hemorrhagic conversion. There was also a suspected tiny subacute infarct within the right centrum semiovale (Figure 6). Despite these findings, which suggest extensive

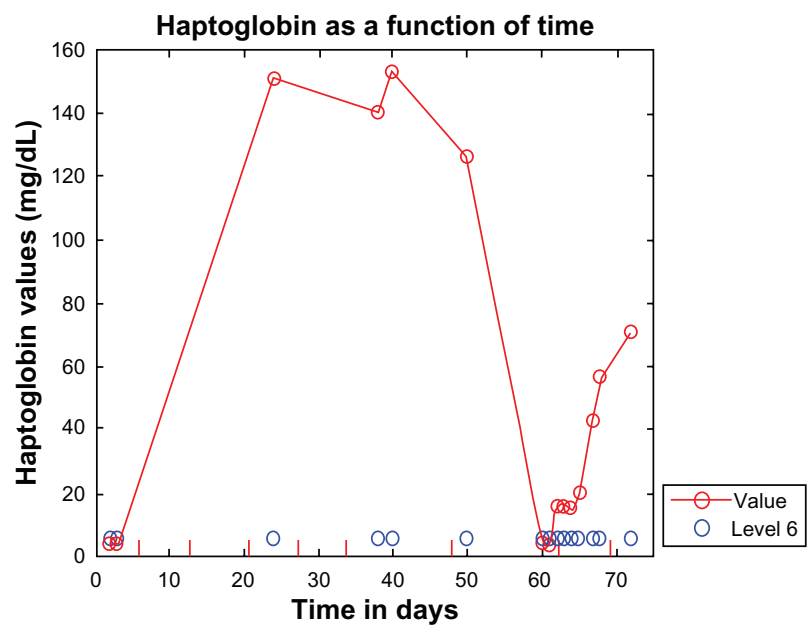

Figure 4 Haptoglobin shown as a function of time (hospital days). Eculizumab administration is denoted by vertical tick marks (hospital days $6,13,20,27,34,48$, and days 63 and 69). Initial haptoglobin was $<6$ (normal range 62-197) mg/dL. Five days after the third dose, haptoglobin was normal and remained normal until the time the seventh dose was due. Haptoglobin then dropped precipitously, indicating an ongoing need for the drug. Haptoglobin did increase again after the seventh dose on day 63 , and later normalized to $7 \mathrm{l} \mathrm{mg} / \mathrm{dL}$ three days after receiving the eighth dose on day 69. 


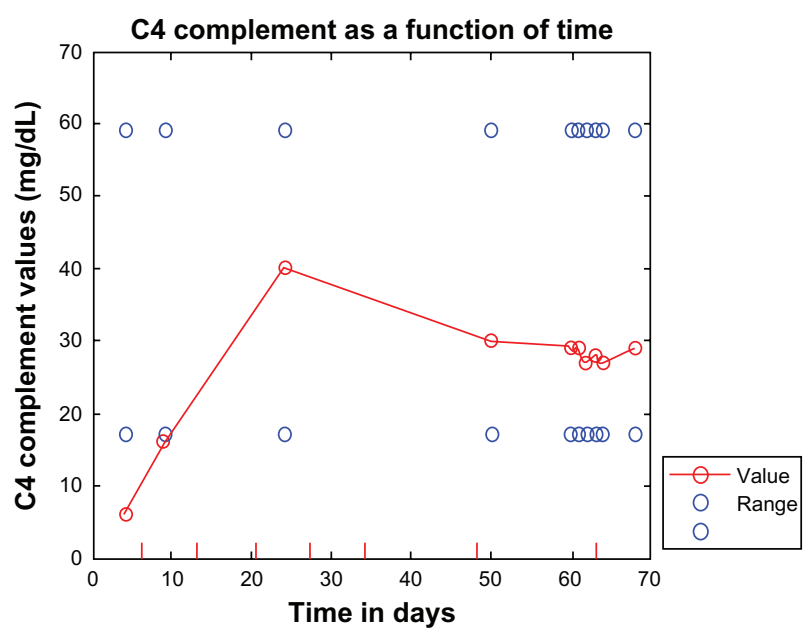

Figure 5 C4 (normal 17-59 mg/dL) shown as a function of time (hospital days). Eculizumab administration is denoted by vertical tick marks (hospital days 6, 13, 20, $27,34,48$, and day 63 ). The day after the second dose of eculizumab on day 13, C4 rose to 16 . Three days after the sixth dose, C4 was normal and remained normal.

thrombotic microangiopathic brain injury, she has recovered neurologically and remains stable.

The dosing schema used in this patient is similar to that used in the clinical trials of eculizumab in atypical hemolytic uremic syndrome. Maintenance therapy, starting on week 5, was planned to be $1200 \mathrm{mg}$ every two weeks. ${ }^{4-6}$ Due to nausea at that dose, the seventh dose was delayed by one day because the patient refused it, but then agreed to a reduced dose of $600 \mathrm{mg}$ weekly. With antiemetic premedications, there was no nausea. Nausea has been reported with eculizumab in up to $16 \%$ of cases. ${ }^{7}$ When the seventh dose was due, laboratory investigations suggested relapsing hemolysis, with haptoglobin $<6 \mathrm{mg} / \mathrm{dL}$, indicating her continued need for the drug. As shown in Figure 4, the haptoglobin increased again after the seventh dose, and normalized to $71 \mathrm{mg} / \mathrm{dL}$ three days after the eighth dose. Her creatinine continued to improve despite the dose modification.

\section{Discussion}

\section{Atypical versus typical hemolytic uremic syndrome}

The annual incidence of Shiga toxin-positive hemolytic uremic syndrome is two cases per 100,000. The annual incidence of hemolytic uremic syndrome in adults in our patient's age group, ie, 50-59 years, is approximately 0.5 cases per $100,000 .^{8,9}$ Shiga toxin-positive hemolytic uremic syndrome primarily affects children. In children under five years, the annual incidence reaches 6.1 cases per 100,000 . Of cases in children, $90 \%$ are caused by Shiga-like toxin-producing E. coli or Shiga toxin-producing Shigella dysenteriae. This form of hemolytic uremic syndrome, classified as typical or enteropathic hemolytic uremic syndrome, involves diarrhea followed by acute renal failure. Streptococcus pneumoniae also causes a severe form of hemolytic uremic syndrome unrelated to Shiga or Shiga-like toxin-producing organisms. ${ }^{8,9}$

A minority of hemolytic uremic syndrome cases, generally unrelated to Shiga /Shiga-like toxin or S. pneumoniae, are termed atypical hemolytic uremic syndrome. Atypical hemolytic uremic syndrome accounts for approximately $10 \%$ of pediatric hemolytic uremic syndrome cases and the

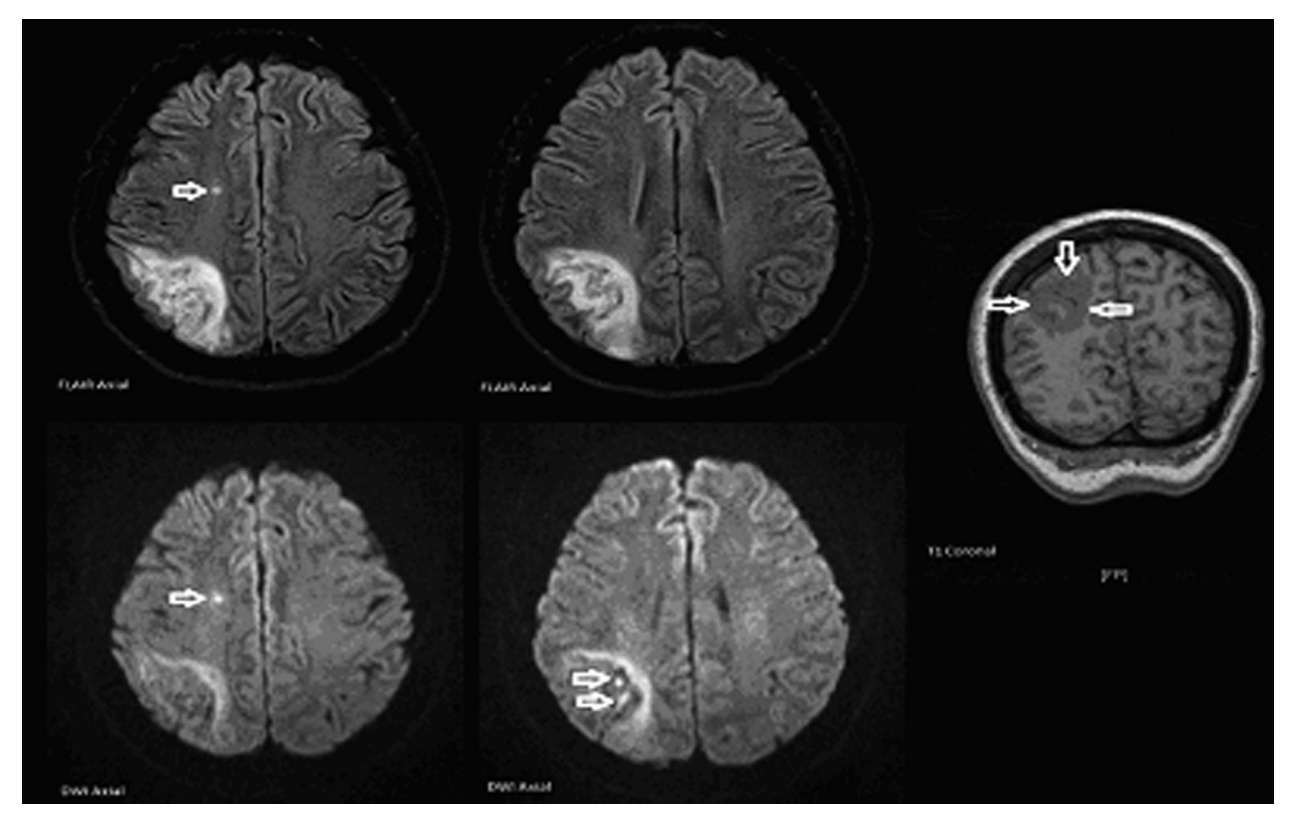

Figure 6 Magnetic resonance imaging scan findings. 
majority of adult cases..$^{8-10}$ The prevalence and incidence of atypical hemolytic uremic syndrome, a rare condition, is not well established. ${ }^{11}$ The estimated prevalence of atypical hemolytic uremic syndrome in European children under 18 years is approximately 3.3 per million. ${ }^{12}$ The prevalence in adults may be lower. The estimated overall annual incidence of atypical hemolytic uremic syndrome in the US is one in $500,000 .^{13}$

The pathogenesis of atypical hemolytic uremic syndrome involves genetic or acquired alternative complement pathway regulator deficiencies. ${ }^{8-10}$ In atypical hemolytic uremic syndrome, reduced C3 levels may sometimes be observed, further suggesting uncontrolled complement activation and consumption. Because the alternative complement pathway convertase requires cleavage of $\mathrm{C} 3$ and not $\mathrm{C} 4$, low serum $\mathrm{C} 3$ and normal $\mathrm{C} 4$ suggest selective alternative pathway activation. ${ }^{9}, 14$

In typical hemolytic uremic syndrome, renal function is preserved in $75 \%$ of cases, whereas the majority of patients with atypical hemolytic uremic syndrome develop end-stage renal disease or die. ${ }^{8,9,15,16}$ In the acute phase of hemolytic uremic syndrome, mortality is $3 \%-5 \%$. In contrast, atypical hemolytic uremic syndrome has a significantly worse prognosis, with a mortality of up to $25 \%$ in the acute phase. ${ }^{8,9,14}$

Under $20 \%$ of atypical hemolytic uremic syndrome cases are familial. The rest are considered to be sporadic, and are triggered by a variety of pathologies, including human immunodeficiency virus, chemotherapeutic agents, immunotherapeutic agents, antiplatelet agents, cancer, transplant, and pregnancy. In the post-transplant setting, de novo hemolytic uremic syndrome has been associated with calcineurin inhibitors (cyclosporine [5\%-15\%], tacrolimus [1\%]), and humoral rejection. Approximately $50 \%$ of sporadic cases of atypical hemolytic uremic syndrome show no obvious cause and are considered idiopathic. ${ }^{8,9}$

Prodromic diarrhea has often been used to distinguish typical from atypical hemolytic uremic syndrome. Our case of atypical hemolytic uremic syndrome demonstrates how prodromic diarrhea alone cannot be used to distinguish these two conditions. Approximately $20 \%-30 \%$ of atypical hemolytic uremic syndrome cases involve prodromic diarrhea. ${ }^{14}$ Although our patient's stool studies did not reveal any Shiga or Shiga-like toxin-producing organisms, these organisms are not always recoverable from the stool of patients with hemolytic uremic syndrome. Such organisms may trigger hemolytic uremic syndrome in patients with pre-existing complement dysregulation. ${ }^{10}$
In our patient, thrombotic microangiopathy resulting in ischemic bowel could have caused her diarrhea and hematochezia. In the absence of Shiga toxin in stool, assessing a patient's complement system function is appropriate. ${ }^{14}$ Our patient was on etanercept and an estrogen patch, both of which are potential endothelial offenders and precipitants of atypical hemolytic uremic syndrome/ complement dysregulation.

\section{Eculizumab in atypical hemolytic uremic syndrome}

Eculizumab, a high-affinity humanized monoclonal anti-C5 antibody, blocks terminal complement activity by binding to and blocking cleavage of the terminal complement protein, $\mathrm{C} 5$, into its proinflammatory, prothrombotic, and lytic products: $\mathrm{C} 5 \mathrm{a}$ and the cytotoxic membrane-attack complex, C5b-9. Upstream components of complement, most notably $\mathrm{C} 3 \mathrm{~b}$, are left intact. ${ }^{3,17,18} \mathrm{In}$ several case reports, eculizumab has been reported to be safe and effective in atypical hemolytic uremic syndrome, a condition of chronic systemic uncontrolled complement activation. $^{3,17-27}$

The doses used in the current clinical trials of eculizumab in atypical hemolytic uremic syndrome are higher than the doses used in paroxysmal nocturnal hemoglobinuria. ${ }^{4-7}$ This seems reasonable, given the risk of multiorgan failure as a result of thrombotic microangiopathy in atypical hemolytic uremic syndrome.

Although no prospective controlled trials have been conducted, plasma exchange has historically been used to manage patients with atypical hemolytic uremic syndrome. ${ }^{28}$ Plasma exchange generally must be used with high frequency to stabilize the condition, ${ }^{24}$ and often loses efficacy. ${ }^{16}$ Additionally, plasma intervention has been associated with a $26 \%$ chance of major complications. ${ }^{29}$ Furthermore, compared with eculizumab, plasma exchange does not specifically treat the underlying defect in complement pathway regulation..$^{28}$ Thus, eculizumab may prove to be more efficacious in the long term, and further investigation is warranted.

Complete and sustained terminal complement inhibition is considered necessary for adequate control of atypical hemolytic uremic syndrome. The optimal duration and frequency of therapy remains to be determined and varies among patients. For example, Nurnberger et al described an adult with relapsed atypical hemolytic uremic syndrome, despite a second kidney transplant, who maintained remission after only one dose of eculizumab $600 \mathrm{mg} .{ }^{17}$ Unfortunately, 
21 months later, this patient suffered allograft loss after failing eculizumab retreatment for recurrence of atypical hemolytic uremic syndrome. ${ }^{14}$ In contrast, Gruppo et al described an infant with atypical hemolytic uremic syndrome refractory to plasma therapy, who required maintenance eculizumab to sustain remission. The patient received eculizumab $300 \mathrm{mg}$ weekly in three doses. Remission occurred 10 days after the first dose, and lasted for at least four months on maintenance eculizumab $600 \mathrm{mg}$ every two weeks. ${ }^{25}$

Mache et al discussed eculizumab dosing for an adolescent with plasmapheresis-refractory atypical hemolytic uremic syndrome. An initial $600 \mathrm{mg}$ was administered, and platelets normalized in three days, haptoglobin normalized in five days, and renal function improved. Atypical hemolytic uremic syndrome relapsed two weeks after recovery of complement-mediated hemolytic activity. Treatment with $600 \mathrm{mg}$ was repeated three times in six days, with only minor, transient improvement in renal function, and hemodialysis was ultimately required. ${ }^{1}$

Chatelet et al described the consequences of delaying the ninth dose of eculizumab in a renal transplant patient with recurrent atypical hemolytic uremic syndrome. The patient was on a maintenance dose of $1200 \mathrm{mg}$ every 14 days after receiving $900 \mathrm{mg}$ weekly for four doses. They reported that a six-day delay in administering the ninth dose resulted in recurrent hemolysis and deterioration of transplant function, necessitating resumption of eculizumab. The authors resumed eculizumab as permanent therapy for their patient, hypothesizing that the delayed dose caused complement breakthrough and relapse of thrombotic microangiopathy. ${ }^{20}$ Importantly, after 17 months of eculizumab therapy, acute thrombotic microangiopathy and hemolysis were controlled, renal function was maintained, and the need for blood transfusion decreased. ${ }^{2}$

\section{Genetic abnormalities associated with atypical hemolytic uremic syndrome}

Around $60 \%$ of atypical hemolytic uremic syndrome cases, both familial and sporadic, have been linked to genetic mutations in complement regulatory proteins in the alternative complement pathway or autoantibodies to complement factor $\mathrm{H}(\mathrm{CFH}) .{ }^{8,9}$ Consequently, we ordered genetic and antibody tests for our patient and the results are still pending. These tests are not required for diagnosis of atypical hemolytic uremic syndrome. Information on genetic abnormalities of the complement system as it relates to atypical hemolytic uremic syndrome can be obtained from two comprehensive papers by Norris and Remuzzi. ${ }^{8,9}$
Genetic testing is important in the care of patients with atypical hemolytic uremic syndrome because prognosis can vary depending on the gene mutations involved. For example, CFH mutations, with cardiac complications in $20 \%$ of cases, are associated with a worse long-term survival. $\mathrm{CFH}$ mutations are associated with a 10-year survival rate of only $40 \%-50 \%$ compared with cases of anti-CFH antibodies, $C F I$ mutations, and $C 3$ mutations, which have a 10 -year survival rate of around $80 \%-90 \%$. Furthermore, genetic testing provides the clinician with prognostic and predictive information about the disease course, treatment response, and long-term transplant outcome. ${ }^{8,9}$

Healthy carriers of $C F H, M C P$, or $C F I$ mutations have an approximately $50 \%$ chance of developing atypical hemolytic uremic syndrome. This suggests that complement gene mutations are predisposing rather than causative, and expression of atypical hemolytic uremic syndrome is dependent upon other genetic or environmental factors. ${ }^{1,8,9}$

Genetic testing is relevant in unaffected family members because mutation carriers can be monitored during conditions triggering complement activation, such as infections, drug exposures (including oral contraceptives), and pregnancy. Infections directly trigger the complement system. Drugs and pregnancy indirectly trigger complement activation by causing endothelial insult. , $^{8}$

Genetic testing is important for patients with atypical hemolytic uremic syndrome being considered for renal transplant. While outcomes of renal transplant vary depending on the genetic mutations, overall, there is a $50 \%$ rate of atypical hemolytic uremic syndrome recurrence in the allograft. ${ }^{8,9}$

Mutations of $C F H, C F I, C F B$ and $C 3$ are associated with post-transplant recurrence. This is because the mutations give rise to abnormalities in circulating proteins which mostly come from the liver, and thus can exist even after kidney transplant. 8,9

In total, $80 \%-90 \%$ of patients with $C F H$ and $C F I$ mutations had allograft relapse following transplant. Consequently, simultaneous liver-kidney transplant has been recommended for these patients. ${ }^{89}$ However, Zuber et al described pre-emptive plasma therapy and eculizumab as promising treatments which may reduce the need for simultaneous liver-kidney transplant. ${ }^{14}$

For patients with $C 3$ mutations undergoing kidney transplant, there is a $40 \%-50 \%$ risk of recurrence in the allograft. However, due to significant extrahepatic synthesis of $\mathrm{C} 3$, combined kidney-liver transplant is not recommended. Furthermore, for patients with $C F B$ mutations, isolated 
kidney transplant and combined kidney-liver transplant is not recommended, because those patients are at increased surgical risk due to severe vascular disease. ${ }^{8,9}$

\section{Conclusion}

Eculizumab can safely reverse neurologic impairment and eliminate the need for dialysis in severe atypical hemolytic uremic syndrome. Because clinical trials are underway, the optimal duration of treatment with eculizumab remains to be determined. Although genetic abnormalities in complement regulatory factors or anti-CFH antibodies have been identified in approximately $60 \%$ of cases, genetic and antibody testing is not required for diagnosis of atypical hemolytic uremic syndrome. ${ }^{8,9}$ Because $20 \%-30 \%$ of atypical hemolytic uremic syndrome cases present with diarrhea, this presenting symptom alone cannot be used to distinguish atypical and enteropathic hemolytic uremic syndrome. ${ }^{14}$

\section{Acknowledgment}

The authors would like to acknowledge Dr Camille Bedrosian for her important recommendations on the treatment of atypical hemolytic uremic syndrome.

\section{Disclosure}

The authors report no conflicts of interest in this work.

\section{References}

1. Mache CJ, Acham-Roschitz B, Fremeaux-Bacchi V, et al. Complement inhibitor eculizumab in atypical hemolytic uremic syndrome. Clin J Am Soc Nephrol. 2009;4(8):1312-1316.

2. Chatelet V, Lobbedez T, Fremeaux-Bacchi V, Ficheux M, Ryckelynck JP, Hurault de Ligny B. Eculizumab: Safety and efficacy after 17 months of treatment in a renal transplant patient with recurrent atypical hemolytic-uremic syndrome: Case report. Transplant Proc. 2010; 42(10):4353-4355.

3. Davin JC, Gracchi V, Bouts A, Groothoff J, Strain L, Goodship T. Maintenance of kidney function following treatment with eculizumab and discontinuation of plasma exchange after a third kidney transplant for atypical hemolytic uremic syndrome associated with a $\mathrm{CFH}$ mutation. Am J Kidney Dis. 2010;55(44):708-711.

4. ClinicalTrials.gov NCT00838513. Available from http://clinicaltrials. gov/ct2/show/NCT00838513?term=nct00838513\&rank=1. Accessed April 7,2010.

5. Muus P, Legendre C, Douglas K, et al. Safety and efficacy of eculizumab in aHUS patients on chronic plasma therapy: Interim analysis from a Phase II trial. Abstract presented at the 43rd annual meeting of the American Society of Nephrology, Denver, CO, November 16-21, 2010. Available from: http://www.abstracts2view.com/asn/view. php?nu=ASN10 L1_157a\&terms. Accessed April 7, 2010.

6. Legendre C, Babu S, Furman R, et al. Safety and efficacy of eculizumab in aHUS patients resistant to plasma therapy: Interim analysis from a Phase II trial. Abstract presented at the 43rd annual meeting of the American Society of Nephrology, Denver, CO, November 16-21, 2010. Available from: http://www.abstracts2view.com/asn/view.php? nu=ASN10L1_157a\&terms. Accessed April 7, 2010.
7. Soliris ${ }^{\circledR}$ (eculizumab). Full prescribing information. Cheshire, CT: Alexion Pharmaceuticals; 2009.

8. Noris M, Remuzzi G. Genetics and genetic testing in hemolytic uremic syndrome/thrombotic thrombocytopenic purpura. Semin Nephrol. 2010; 30(4):395-408.

9. Noris M, Remuzzi G. Atypical hemolytic-uremic syndrome. $N$ Engl J Med. 2009;361(17):1676-1687.

10. Bitzan M, Schaefer F, Reymond D. Treatment of typical (enteropathic) hemolytic uremic syndrome. Semin Thromb Hemost. 2010;36(6): 594-610.

11. Pollock KG, Young D, Beattie TJ, Todd WT. Clinical surveillance of thrombotic microangiopathies in Scotland, 2003-2005. Epidemiol Infect. 2008;136(1):115-121.

12. Zimmerhack1 LB, Besbas N, Jungraithmayr T, et al. Epidemiology, clinical presentation, and pathophysiology of atypical and recurrent hemolytic uremic syndrome. Semin Thromb Hemost. 2006;32(2): 113-120.

13. Genetics Home Reference. Atypical hemolytic-uremic syndrome. Available from: http://ghr.nlm.nih.gov/condition/atypical-hemolyticuremic-syndrome. Accessed March 25, 2011.

14. Zuber J, Le Quintrec M, Sberro-Soussan R, Loirat C, Fremeaux-Bacchi V, Legendre C. New insights into postrenal transplant hemolytic uremic syndrome. Nat Rev Nephrol. 2011;7(1):23-35.

15. Loirat C, Noris M, Fremeaux-Bacchi V. Complement and the atypical hemolytic uremic syndrome in children. Pediatr Nephrol. 2008;23(11): 1957-1972.

16. Noris M, Caprioli J, Bresin E, et al. Relative role of genetic complement abnormalities in sporadic and familial aHUS and their impact on clinical phenotype. Clin J Am Soc Nephrol. 2010;5(10):184-189.

17. Nurnberger J, Phillip T, Witzke O, et al. Eculizumab for atypical hemolytic-uremic syndrome. $N$ Engl J Med. 2009;360(5):542-544.

18. Dmytrijuk A, Robie-Suh K, Cohen MH, Rieves D, Weiss K, Pazdur R. FDA report: Eculizumab (Soliris) for the treatment of patients with paroxymal nocturnal hemoglobinuria. Oncologist. 2008;13(9): 993-1000.

19. Zimmerhackl LB, Hofer J, Cortina G, et al. Prophylactic eculizumab after renal transplantation in atypical hemolytic-uremic syndrome. N Engl J Med. 2010;362(18):1746-1748.

20. Chatelet V, Fremeaux-Bacchi V, Lobbedez T, Ficheux M, Hurault de Ligny B. Safety and long-term efficacy of eculizumab in a renal transplant patient with recurrent atypical hemolytic-uremic syndrome. Am J Transplant. 2009;9(11):2644-2645.

21. Ardissino G, Testa S, Paglialonga F, et al. Remission of plasmaresistant atypical hemolytic uremic syndrome relapse on kidney graft with eculizumab. Abstract presented at the Second International Conference on HUS-MPGN-PNH, June 13-15, 2010, Innsbruck, Austria.

22. Haffner H, Hofer J, Zimmerhackl LB, Pohl M. Effective eculizumab therapy of familiar atypical HUS in a 4 year old patient. Abstract presented at the Second International Conference on HUS-MPGN-PNH, June 13-15, 2010, Innsbruck, Austria.

23. Lapayraque AL, Phan V, Clermont MJ, et al. Effectiveness of eculizumab in a plasma infusion dependent patient with atypical haemolytic and uremic syndrome (aHUS) associated with heterozygous combined de novo mutation in factor $\mathrm{H}$ gene. Abstract presented at the Second International Conference on HUS-MPGN-PNH, June 13-15, 2010, Innsbruck, Austria.

24. Tschumi S, Bucher BS, Sparta G, et al. Eculizumab in atypical hemolytic uremic syndrome: Long-term clinical course and histological findings. Abstract presented at the Second International Conference on HUSMPGN-PNH, June 13-15, 2010, Innsbruck, Austria.

25. Gruppo RA, Rother RP. Eculizumab for congenital atypical hemolyticuremic syndrome. N Engl J Med. 2009;360(5):544-546.

26. Larreal CF, Cofan F, Oppenheimer F, Campistol JM, Escolar G, Lozano M. Efficacy of eculizumab in the treatment of recurrent atypical hemolytic-uremic syndrome after renal transplantation. Transplantation. 2010;89(7):903-904. 
27. Legault DJ, Boelkins MR. Successful treatment of aHUS recurrence and arrest of plasma exchange resistant TMA post-renal transplantation with the terminal complement inhibitor eculizumab. Abstract presented at the 51st annual meeting of the American Society of Hematology, December 5-8, 2009, New Orleans, LA.
28. Loirat C, Garnier A, Sellier-Leclerc AL, Kwon T. Plasmatherapy in atypical hemolytic uremic syndrome. Semin Thromb Hemost. 2010; 36(6):673-681.

29. George JN. How I treat patients with thrombotic thrombocytopenic purpura. Blood. 2010;116(20):4060-4069.

\section{Publish your work in this journal}

Clinical Pharmacology: Advances and Applications is an international, peer-reviewed, open access journal publishing original research, reports,

reviews and commentaries on all areas of drug experience in humans.

The manuscript management system is completely online and includes

a very quick and fair peer-review system, which is all easy to use.
Visit http://www.dovepress.com/testimonials.php to read real quotes from published authors. 